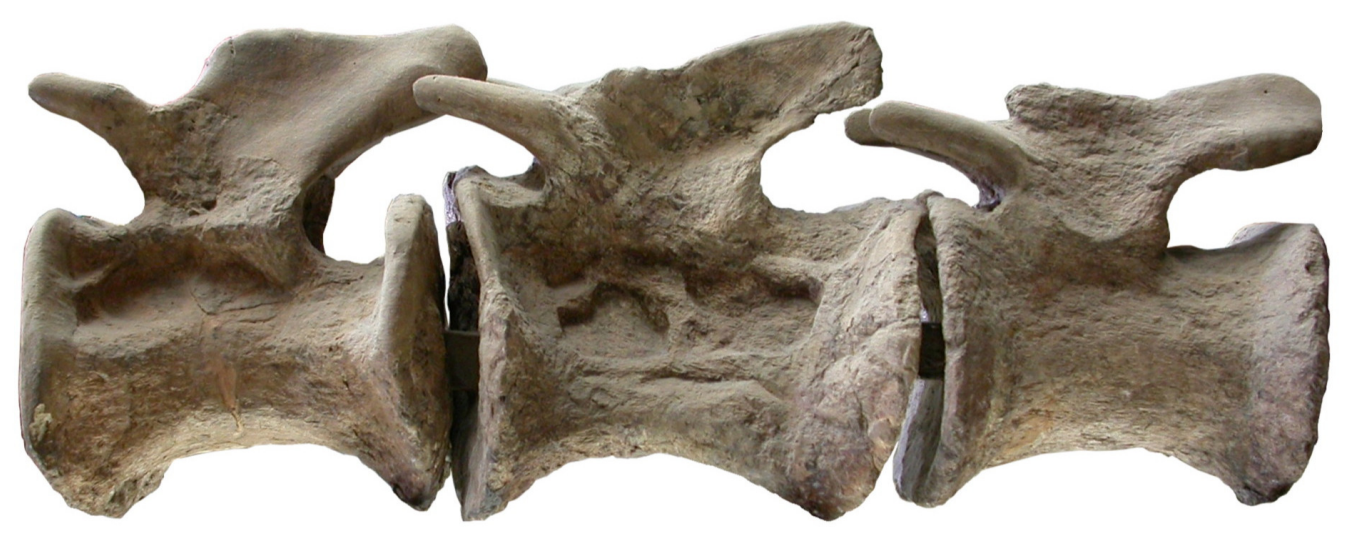

\title{
Why is vertebral pneumaticity in sauropod dinosaurs so variable?
}

\author{
Mike Taylor ${ }^{1}$, Mathew Wedel $^{2}$ \\ 1 University of Bristol \\ 2 Western University of Health Sciences
}

Funding: The author(s) received no specific funding for this work.

Potential competing interests: The author(s) declared that no potential competing interests exist.

\section{Abstract}

The vertebrae of sauropod dinosaurs have distinctive and complex pneumatic features including fossae and foramina in the sides of their centra. These vary between individuals, serially within individuals, and even between the left and right sides of single vertebrae. This presents a conundrum because bone is usually the least variable material in the vertebrate body. Blood vessels, however, are much more labile, as can be seen in the varied occurrence of vascular foramina in the vertebrae not only of sauropods, but also of birds, crocodilians and mammals. Vascular variation arises in part from the ontogeny of vertebrae, which in their embryonic state are vascularised from within the neural canal: the hand-off from these vessels to others which penetrate from outside is not always completed. In birds, pneumatizing diverticula enter the vertebrae alongside blood vessels, in the vascular foramina that they form, before excavating the surrounding bone into larger pneumatic foramina. We propose that the same was true in sauropods, and that variation of vascularization directly caused variation of pneumatization. In most vertebrae, a single vascular foramen carries both artery and vein, but occasionally these vessels separate and each forms a separate foramen. This explains why in rare cases individual sauropod vertebrae have two pneumatic cavities on a single side: each arises from the vascular foramen formed by either artery or vein. 


\section{Introduction}

The vertebrae of sauropod dinosaurs are distinctive not only because of their size but also because they have complex pneumatic features. These include fossae and foramina, in both the centrum and neural arch; and laminae connecting landmarks such as the zygapophyses, diapophyses and parapophyses (Wedel 2003). For this reason, sauropod vertebrae are unusually diagnostic and are frequently used in species determination (McIntosh 1990).

Bone is generally the material that varies the least between individuals of a species, with muscle, nerves and especially blood vessels being more prone to variation (Berger 1956:435-439, Moore et al. 2010:11). However, while pneumatic features of sauropod vertebrae can be characteristic of a species, genus or clade, they are also highly variable: not only between individuals, but also along the column of an individual (e.g. Diplodocus carnegii, Hatcher 1901:plates 3 and 7), and even sometimes between the sides of a single vertebra. Examples of the latter include the single vertebra that is the Xenoposeidon proneneukos holotype: Taylor and Naish (2007:1552; Figure 1); and the sequence of vertebrae in the tail of Giraffatitan brancai MB.R.5000 (Wedel and Taylor 2013:5-7 and figures 4 and 5). In contrast, the vertebrae of mammals, non-dinosaurian reptiles and even other dinosaurs are much more uniform, exhibiting less individual, serial and bilateral variation. Why are sauropod vertebrae so much more variable?
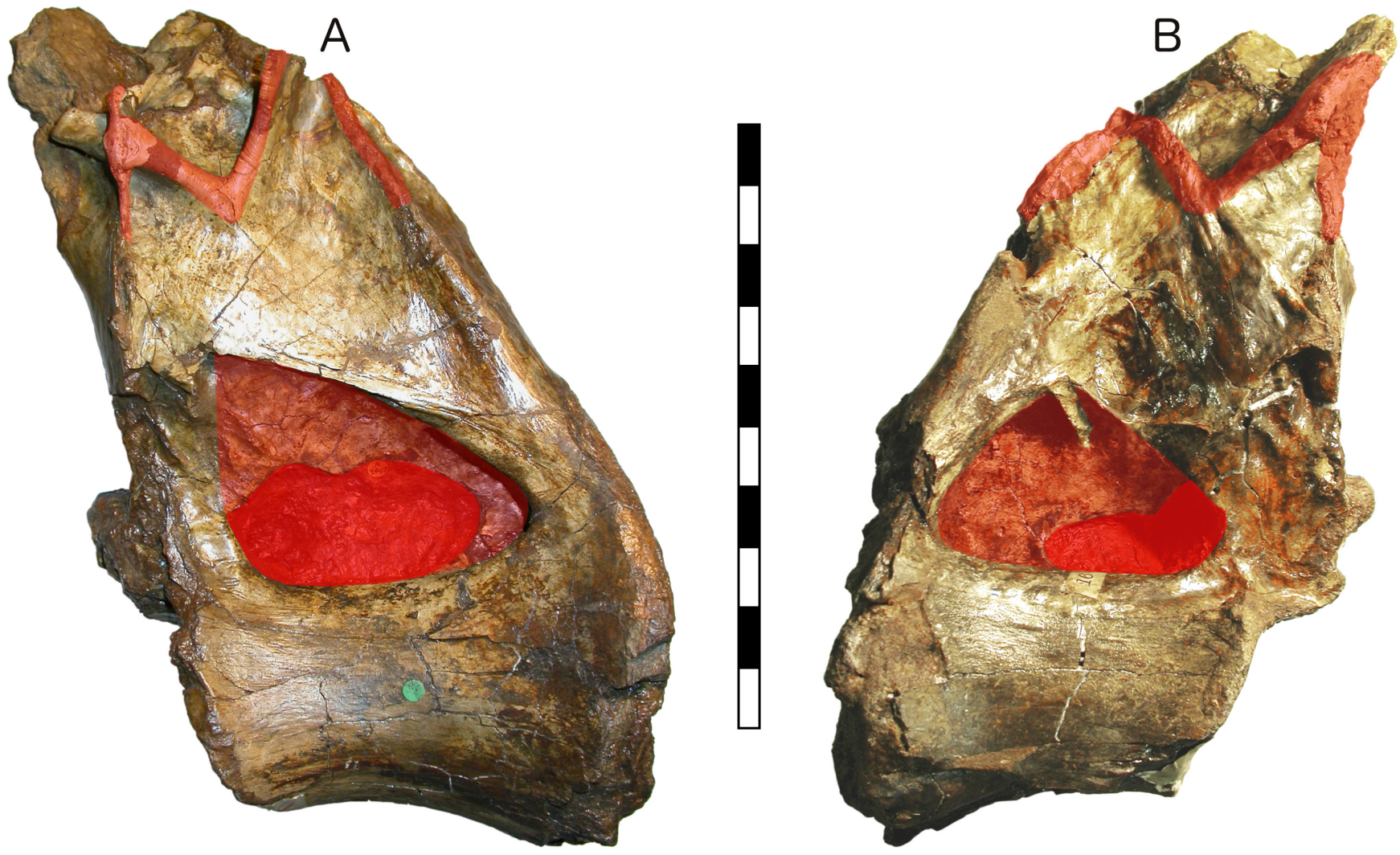

Figure 1. NHMUK PV R2095, the holotype and only known vertebra of the rebbachisaurid sauropod Xenoposeidon proneneukos. A: left lateral view. B: right lateral view. Pneumatic fossa and "M"-shaped complex of laminae highlighted in pale red; pneumatic foramina within fossae highlighted in deep red. Note that, while the general outline of the pneumatic features is the same on both sides, there are numerous differences in detail: the fossae and their contained foramina are different shapes, the fossa on the right contains an accessory lamina, the "M" is better defined on the left, etc. Scale bar $=20 \mathrm{~cm}$. After Taylor (2018:figure 1). 


\section{Analysis}

It has been generally assumed that variation in pneumatic features is essentially random: as Witmer (1997:64) wrote of the antorbital paranasal sinus in archosaurs, "pneumatic diverticula are viewed simply as opportunistic pneumatizing machines, resorbing as much bone as possible within the constraints imposed by local biomechanical loading regimes". However, here we will develop another explanation.

Bremer (1940:200) demonstrated that in extant birds, developing diverticula follow blood vessels as they radiate through the body: "Into this loose tissue, along the vein, the air sac [i.e. diverticulum in modern usage] finally grows in the form of a long tube ... The actual entrance of the air sac into the main marrow cavity is effected at first at the internal opening of the vein". O'Connor (2006:1208-1209) confirmed that "vascular injection studies on birds with pneumatic postcrania reveal that nutrient vessels share (i.e., co-occupy) foramina with pneumatic diverticula to gain access to the medullary space". It is parsimonious to assume the same was true in sauropods.

But vascularization of vertebrae is itself highly variable, and it is common for the pattern of vertebrae with and without external vascular foramina to be random. For example, in a juvenile specimen of the crocodilian Tomistoma, only about half of the first 13 caudal vertebrae have vascular foramina on each side, and they are not the same vertebrae on each side (Figure 2). 


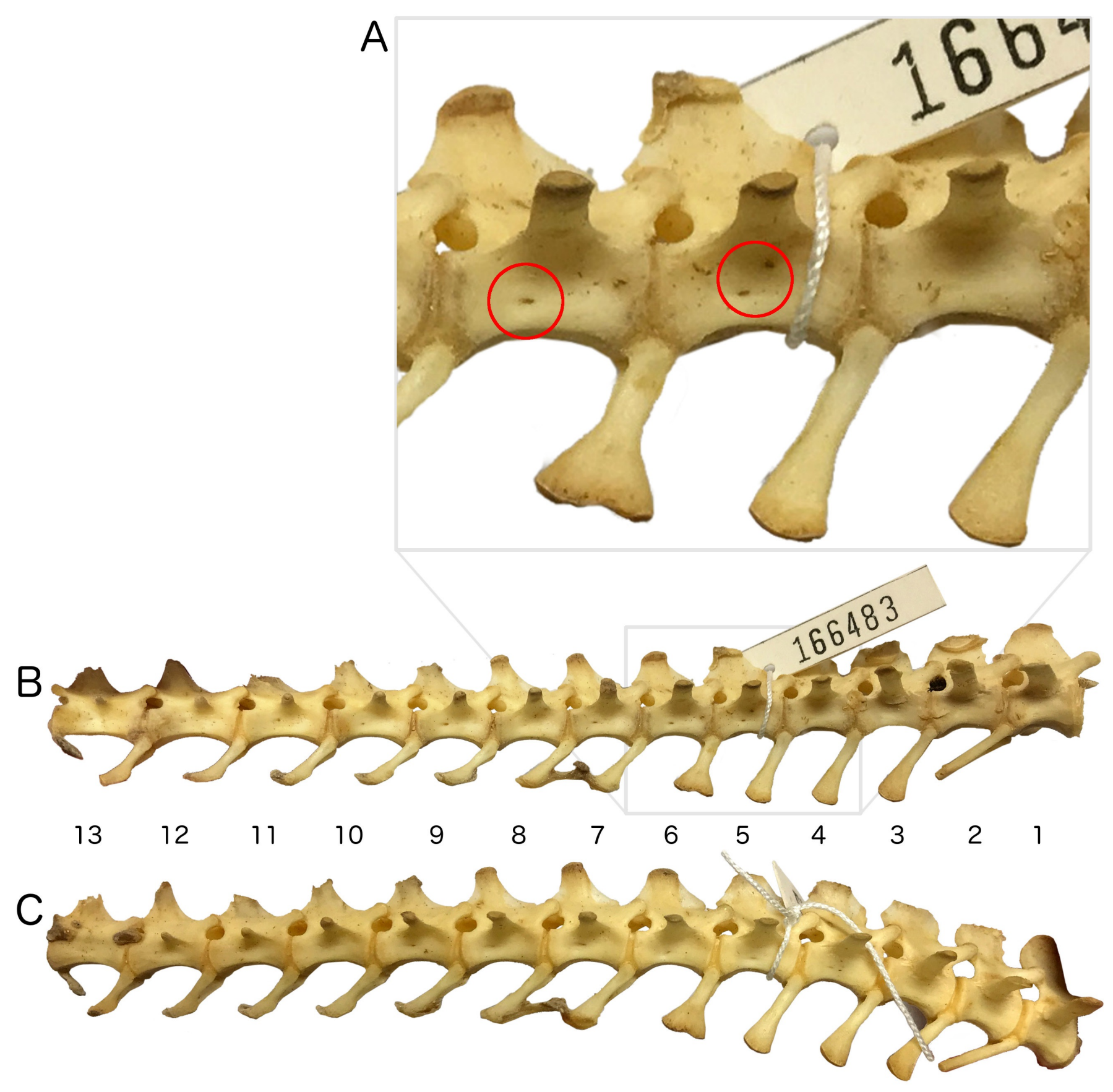

Figure 2. Proximal tail skeleton (first 13 caudal vertebrate) of LACM Herpetology 166483, a juvenile specimen of the false gharial Tomistoma schlegelii. A: close-up of caudal vertebrae 4-6 in right lateral view, red circles highlighting vascular foramina: none in Ca4, two in Ca5 and one in Ca6. B: right lateral view. C: left lateral view. In right lateral view, vascular foramina are apparent in the centra of caudal vertebrae 5-7 and 9-11; they are absent or too small to make out in vertebrae 1-4, 8 and 12-13. In left lateral view, vascular foramina are apparent in the centra of caudal vertebrae 4-7 and 9; they are absent or too small to make out in vertebrae 1-3, 8, and 10-13. Caudal centra 5-7 and 9 are therefore vascularised from both sides; 4 and 10-11 from one side only; and 1-3, 8 and 12-13 not at all.

In sauropods, too, vascularization is variable along the vertebral sequence (Figure 3) and between sides of an individual vertebra. 


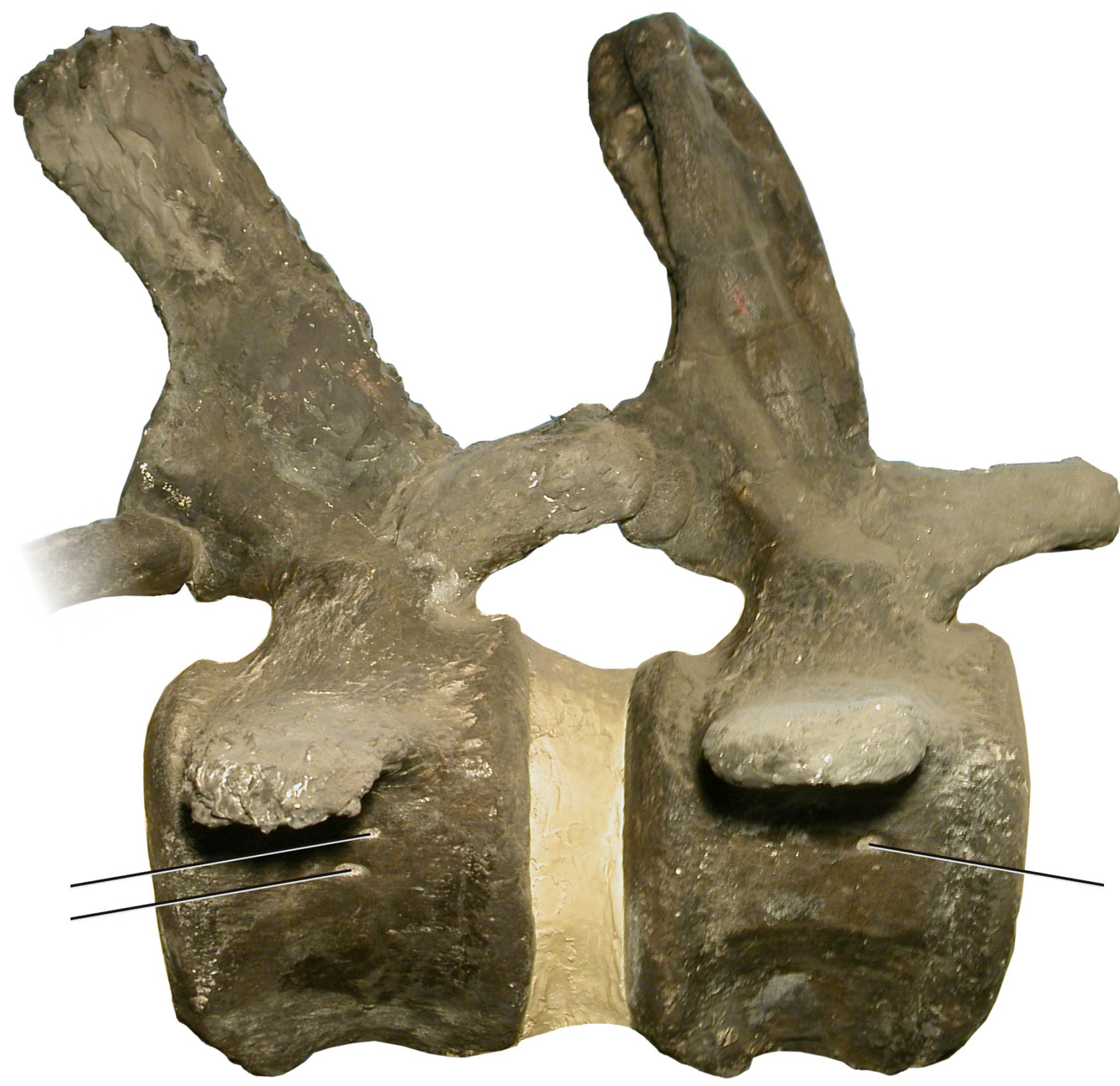

Figure 3. Brontosaurus excelsus holotype YPM 1980, caudal vertebrae 7 and 8 in right lateral view. Lines indicate location of foramina. Caudal 7 , like most of the sequence, has a single vascular foramen on the right side of its centrum, but caudal 8 has two; others, including caudal 1 , have none.

Why is vascularization so variable?

The ontogenetic development of vertebral vasculature is a complex process. Early in embryonic development, the spinal cord is much larger than the vertebrae. Arteries serve the cord first; then as the notochord segments are replaced by the cartilaginous anlagen of the vertebrae, branches of the medullary arteries tunnel into the cartilage and support the growth of the vertebrae (Amato 1959). All the blood supply to developing vertebrae therefore comes from inside the neural canal. Branches of the segmental arteries subsequently penetrate the vertebrae from the outside, and anastomotic connections 
develop inside the vertebra, connecting the internal and external systems (see example in Cramer 2014:figure 2.4).

As the growth of the vertebrae outpaces that of the cord, there is a handoff of arterial supply from the original medullary arteries that serve the cord to the secondary, external arteries: foramina inside the neural canal shrink with age, while those on the external surface of the vertebra enlarge (Smuts 1975:35). However, this handoff is not always completed, and asymmetric arterial supply is common (Smuts 1975). Consequently centra frequently lack an external vascular foramen on one or both sides. This is not a problem as the medullary arteries can provide the necessary blood supply, but in sauropods the absence of such external foramina means there is no point of entry for a diverticulum that otherwise would subsequently produce pneumatic cavities in the side of the bone.

In summary, since external pneumatic cavities follow vascularization of the outer wall, and the latter is variable, it follows that pneumatization is also variable, reflecting the variation in the soft tissues that guide its development.

The fossil record provides additional evidence that pneumatic diverticula follow vascular pathways when pneumatizing the postcranial skeleton. Three observations are of particular relevance: 1. in non-avian theropods, the evolution of pneumaticity in dorsal and sacral vertebrae follows a "neural arch first" pattern (Benson et al. 2012); 2. in basal sauropodomorphs (and possibly other archosaurs) deep, complex fossae ventral to the diapophyses and zygapophyses are among the earliest-evolving correlates of pneumatization (Butler et al. 2012, Yates et al. 2012); 3. in juvenile sauropods, well-developed laminae and fossae are present in the neural arches of dorsal vertebrae before all the centra are pneumatized (Melstrom et al. 2016, Hanik et al. 2017). Pneumatization favoured the neural arches over the centra in these cases because developing diverticula followed the large segmental arteries that serve the spinal cord. It is therefore not surprising that the earliest signs of pneumaticity, both ontogenetically and phylogenetically, should be near the neural canal, low on the neural arch.

The morphogenetic rules governing cervical and caudal pneumatization are less clear than in the torso. In the cervical column, the arteries that supply the spinal cord branch from the paired vertebral arteries, which lie alongside the vertebral centra, and this may explain the "centrum first" pattern of pneumatization in the cervical vertebrae of non-avian theropods (Benson et al. 2012). The caudal vertebrae of juvenile diplodocids are less pneumatic than those of adults (Melstrom et al. 2016, Hanik et al. 2017), and in Giraffatitan extensive caudal pneumaticity is present only in large individuals (Wedel and Taylor 2013). These observations suggest that caudal pneumatization in sauropods continued for several years, after the vascular handoff from neural canal arteries to arteries on the external surface of the centrum, possibly explaining the mix of "centrum first" and "arch first" pneumatization observed in sauropod caudal vertebrae.

It is also notable that paired pneumatic fossae or foramina occur lateral or dorsolateral to the neural canal in every archosaurian clade with postcranial pneumaticity (Figure 4). These fossae and foramina occur in taxa with and without lateral cavities in the centra, and with and without laminated neural arches, so they are probably the most consistent osteological correlates of pneumaticity across non-avian ornithodirans. The consistent appearance of vertebral 
pneumaticity in areas adjacent to the neural canal corroborates the hypothesis that segmental spinal arteries were crucial in "piloting" pneumatic diverticula as they developed.
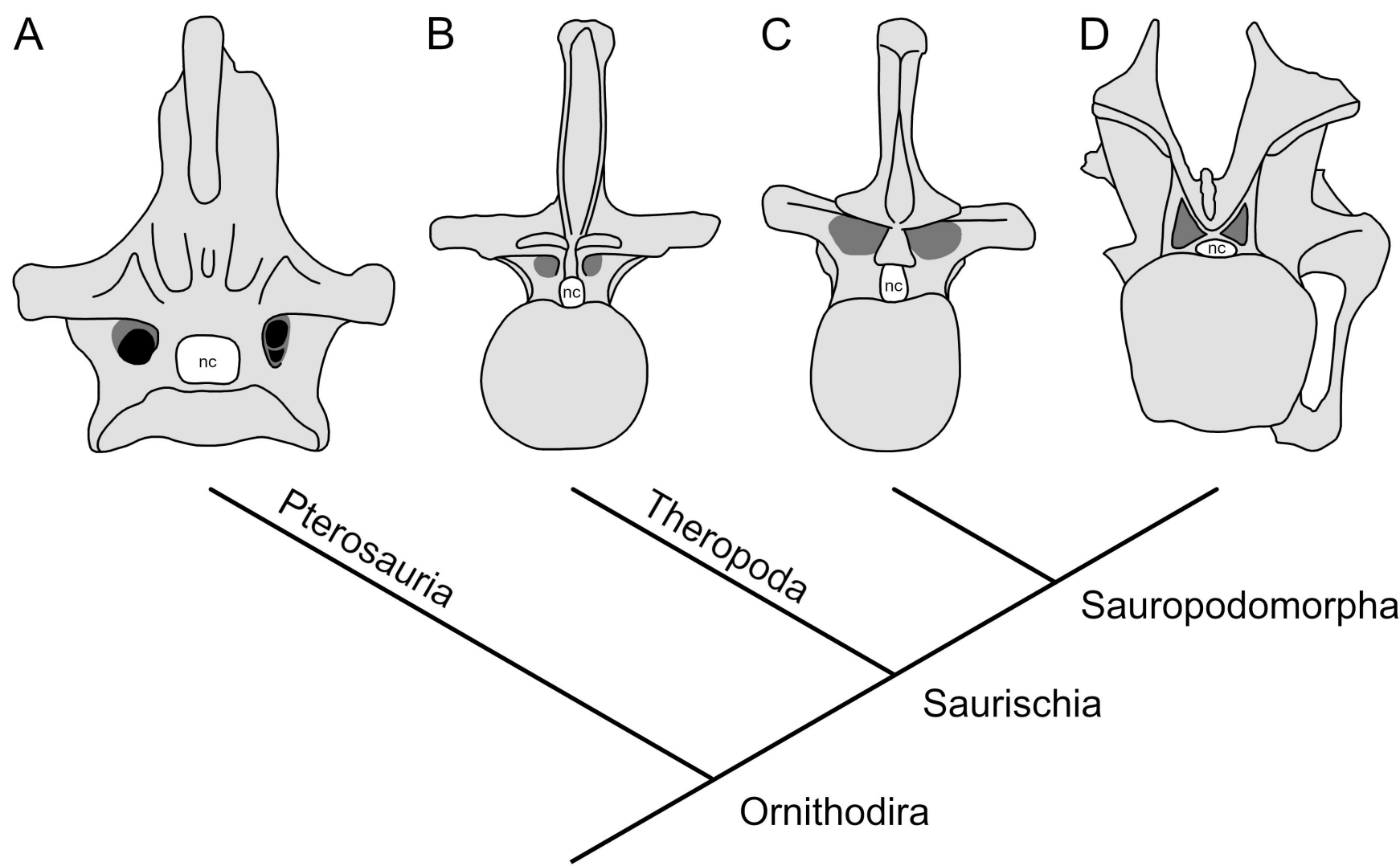

Figure 4. Fossae and foramina adjacent to the neural canal in ornithodiran archosaurs. Fossae are shown in dark grey, foramina in black. Neural canals are labelled "nc". A: Pterosauria, represented by cervical vertebra 9 of Pteranodon sp. YPM 2767 in anterior view (traced from Bennett 2001: figure 42). B: Theropoda, represented by dorsal vertebra 14 of Allosaurus fragilis UUVP 6000 in anterior view (traced from Madsen 1976: plate 23). C: Basal Sauropodomorpha, represented by a posterior dorsal vertebrae of Aardonyx celestae BP/1/6566 in posterior view (traced from Yates et al. 2012: figure 7). D: Neosauropoda, represented by cervical vertebra 5 of Diplodocus carnegii CM 84 in posterior view (traced from Hatcher 1901: plate 6).

\section{Discussion}

As noted by O'Connor (2006:1208), "Whereas arteries and veins often utilize a single nutrient foramen within a given vertebra, occasionally there are separate foramina for each". Similarly, Travan et al. (2015) show that in the cervical vertebrae of humans the transverse foramen, which the vertebral artery and vein pass through, is sometimes double, with the two vessels each passing through its own opening rather than the usual shared opening. (In rare cases, a triple transverse foramen occurs, with the sympathetic nerve plexus passing through a third opening rather than sharing the opening used by one or both blood vessels.) A similar phenomenon can be observed in the tail of the Brontosaurus excelsus holotype YPM 1980, in which the right side of the centrum of caudal 7 has the usual single vascular foramen but that of caudal 8 has two (Figure 3). 
If our hypothesis that pneumatization follows vascularization is correct, then this could explain why there are sometimes two pneumatic fossae on one side of a centrum, for example the left side of caudal 25 of the Giraffatitan brancai tail MB.R.5000 (Figure 5): the two vascular foramina carrying artery and vein were each followed by a pneumatic diverticulum and each developed into a pneumatic fossa.

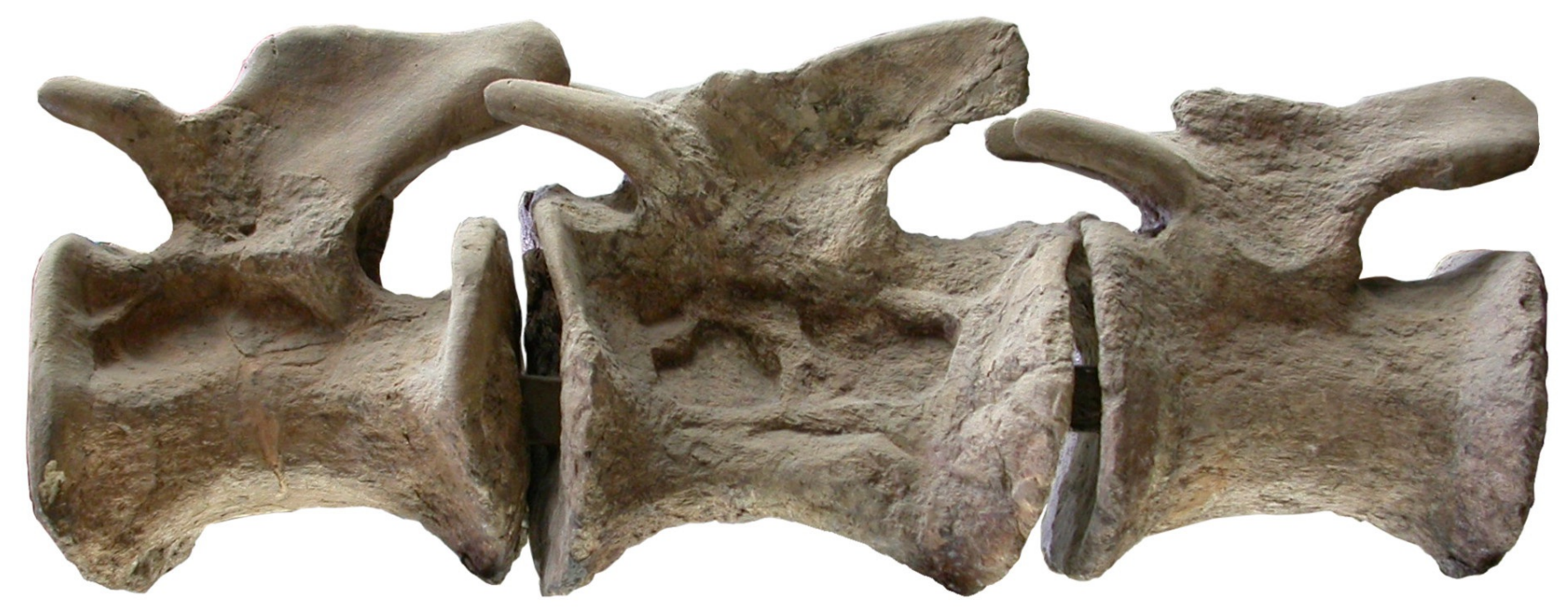

Figure 5. Giraffatitan brancai tail MB.R.5000, part of the mounted skeleton at the Museum für Naturkunde Berlin. Caudal vertebrae 24-26. in left lateral view While caudal 26 has no pneumatic features, caudal 25 has two distinct pneumatic fossae, likely excavated around two distinct vascular foramina carrying an artery and a vein. Caudal 24 is more shallowly excavated than 26, but may also exhibit two separate fossae.

Vascular foramina are rarely if ever seen in sauropod vertebrae that feature pneumatic fossae or foramina. Understandably they do not appear alongside these features, as the cavities were excavated around the blood vessels; but why do vascular foramina not appear within pneumatic cavities?

When a blood vessel enters a bone through a vascular foramen it is still detectable in CT scans as a tunnel through the trabeculae (Butler et al. 2012:figures 3 and 11), but the vessels usually arborize into arterioles and capillaries quickly once they're inside. So before a bone becomes pneumatized by a fossa, the artery has already branched into many small vessels. When the diverticulum subsequently enlarges the vascular foramen into a pneumatic fossa, pneumatization likely excavates the bone around the already-branched arterial tree that existed inside the bone. There should therefore be multiple vascular foramina inside the fossa, representing the multiple branches of the artery - as can be observed in a least some vertebrae of ducks (Figure 6). 


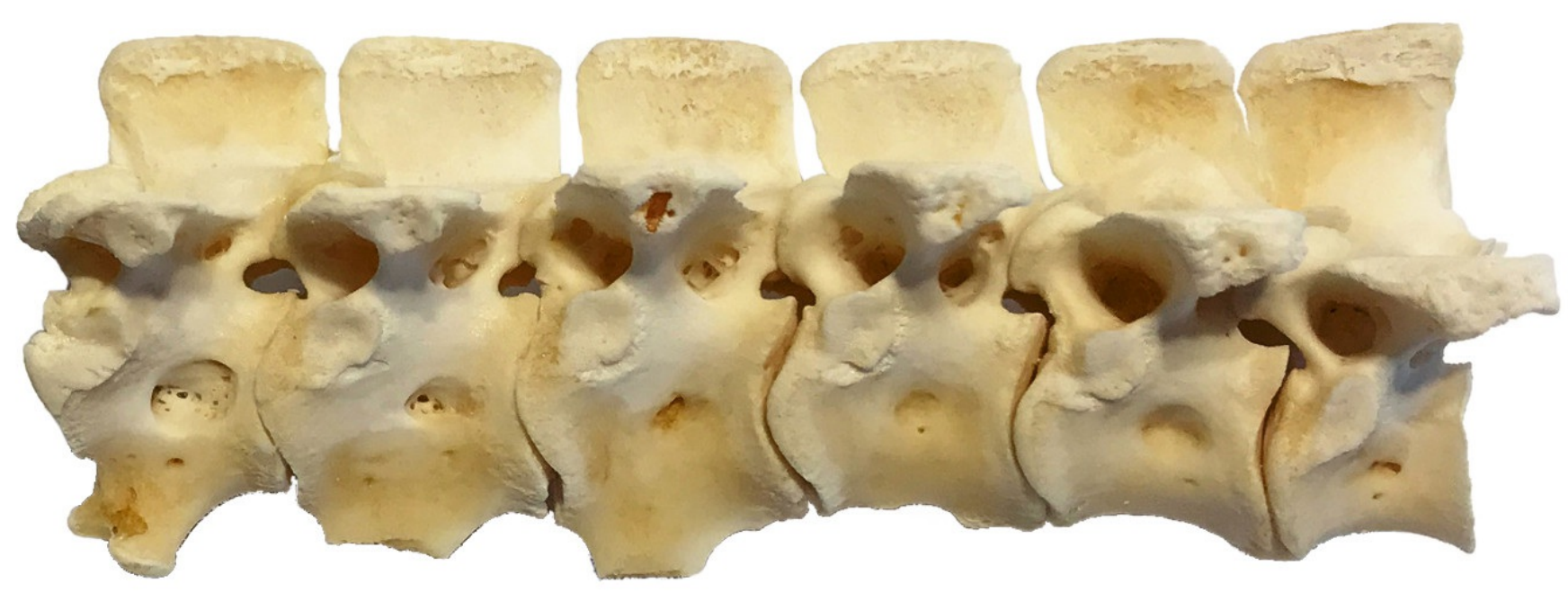

Figure 6. Domestic duck Anas platyrhynchos, dorsal vertebrae 2-7 in left lateral view. Note that the two anteriormost vertebrae (D2 and D3) each have a shallow pneumatic fossa penetrated by numerous small foramina.

However, these foramina will be much smaller than those that remain at the surface of apneumatic vertebrae. They may not be well preserved by fossilization, and even when preserved they will be difficult to spot during fossil preparation especially as pneumatic cavities in large, delicate bones are already difficult to prepare. We recommend that sauropod workers carefully check pneumatic fossae and foramina for evidence of contained vascular foramina.

\section{Conclusion}

In general, bones are the least variable part of a body, followed by muscles, nerves, and finally blood vessels, which are very variable in all vertebrates. Pneumatic fossae and foramina are skeletal features, so they might be expected to fall at the least variable end of the spectrum. But since diverticula follow blood vessels as they develop, the variability of pneumatic features in bones is not a coincidence: the variability of blood vessels causes the variability of diverticula, and of their skeletal traces.

\section{Museum Abbreviations}

- BP - Bernard Price Institute (Palaeontology), University of the Witwatersrand, Johannesburg, South Africa.

- CM - Carnegie Museum of Natural History, Pittsburgh, Pennsylvania, USA.

- LACM - Natural History Museum of Los Angeles County, Los Angeles, California, USA.

- MB.R. - Museum für Naturkunde Berlin, Berlin, Germany (fossil reptile collection).

- NHMUK - Natural History Museum, London, United Kingdom.

- UUVP — Natural History Museum of Utah, Salt Lake City, Utah, USA.

- YPM - Yale Peabody Museum of Natural History, New Haven, Connecticut, USA.

\section{Acknowledgements}


We thank the curators and collections managers at the Museum of Natural History of Los Angeles County, Museum für Naturkunde Berlin, Natural History Museum, London, and Yale Peabody Museum for access to specimens. Mark McMenamin and Fernando E. Novas provided constructive reviews of versions 1 and 2 of this article, yielding the improvements in this current version.

\section{References}

- Amato, V. P., S. Malta and R. Bombelli. 1959. The normal vascular supply of the vertebral column in the growing rabbit. The Journal of Bone and Joint Surgery 41(4):782-795.

- Bennett, S. Christopher, 2001. The osteology and functional morphology of the Late Cretaceous pterosaur Pteranodon, Part I. General description of osteology. Palaeontographica Abteilung A 260:1-112.

- Benson, Roger B., Richard J. Butler, Matthew T. Carrano and Patrick M. O'Connor. 2012. Air-filled postcranial bones in theropod dinosaurs: physiological implications and the "reptile"-bird transition. Biological Reviews 87(1):168-193.

- Berger, Andrew. J. 1956. Anatomical variation and avian anatomy. The Condor 58(6):433-441.

- Bremer, John L. 1940 The pneumatization of the humerus in the common fowl and the associated activity of theelin. The Anatomical Record 77(2):197-211. doi:10.1002/ar.1090770209

- Butler, Richard J., Paul M. Barrett and David J. Gower. 2012. Reassessment of the evidence for postcranial skeletal pneumaticity in Triassic archosaurs, and the early evolution of the avian respiratory system. PLOS ONE 7(3):e34094. doi:10.1371/journal.pone.0034094

- Cramer, Gregory D. 2014. General characteristics of the spine. pp. 15-64 in: Gregory D. Cramer and Susan A. Darby (eds.), Clinical Anatomy of the Spine, Spinal Cord, and ANS, 3rd edition. Elsevier, St. Louis. doi:10.1016/b978-0-32307954-9.00002-5

- Hanik, Gina M., Matthew C. Lamanna and John A. Whitlock. 2017. A juvenile specimen of Barosaurus Marsh, 1890 (Sauropoda: Diplodocidae) from the Upper Jurassic Morrison Formation of Dinosaur National Monument, Utah, USA. Annals of Carnegie Museum 84(3):253-263.

- Hatcher Jonathan B. 1901. Diplodocus (Marsh): its osteology, taxonomy and probable habits, with a restoration of the skeleton. Memoirs of the Carnegie Museum 1:1-63 and plates I-XIII.

- Madsen, Jr., James H. 1976. Allosaurus fragilis: a revised osteology. Utah Geological and Mining Survey Bulletin 109:1-163.

- McIntosh, John S. 1990. Species determination in sauropod dinosaurs with tentative suggestions for their classification. pp. 53-69 in: K. Carpenter and P .J. Currie (eds.), Dinosaur Systematics: Approaches and Perspectives, Cambridge University Press, Cambridge, UK.

- Melstrom, Keegan M., Michael D. D’Emic, Daniel Chure and Jeffrey A. Wilson. 2016. A juvenile sauropod dinosaur from the Late Jurassic of Utah, USA, presents further evidence of an avian style air-sac system. Journal of Vertebrate Paleontology 36(4):e1111898. doi:10.1080/02724634.2016.1111898

- Moore, Keith L., Arthur F. Dalley and Anne M. R. Agur. 2010. Clinically Oriented Anatomy, 6th edition. Lippincott Williams \& Wilkins, Baltimore, 1134 pages.

- O'Connor, Patrick M. 2006. Postcranial pneumaticity: an evaluation of soft-tissue influences on the postcranial skeleton 
and the reconstruction of pulmonary anatomy in archosaurs. Journal of Morphology 267(10):1199-1226. doi:10.1002/jmor.10470.

- Smuts, M. M. S. 1975. The foramina of the cervical vertebrae of the ox, part Il: cervical vertebrae 3-7. Anatomia, Histologia, Embryologia 4(1):24-37.

- Taylor, Michael P. 2018. Xenoposeidon is the earliest known rebbachisaurid sauropod dinosaur. PeerJ 6:e5212. doi: $10.7717 /$ peerj.5212

- Taylor, Michael P., and Darren Naish. 2007. An unusual new neosauropod dinosaur from the Lower Cretaceous Hastings Beds Group of East Sussex, England. Palaeontology 50(6):1547-1564. doi: 10.1111/j.14754983.2007.00728.x

- Travan, Luciana, Paola Saccheri, Giorgia Gregoraci, Chiara Mardegan and Enrico Crivellato. 2015. Normal anatomy and anatomic variants of vascular foramens in the cervical vertebrae: a paleo-osteological study and review of the literature. Anatomical Science International 90:308-323. doi:10.1007/s12565-014-0270-x

- Wedel, Mathew J. 2003. The evolution of vertebral pneumaticity in sauropod dinosaurs. Journal of Vertebrate Paleontology 23:344-357. doi:10.1671/0272-4634(2003)023[0344:TEOVPI]2.0.CO;2

- Wedel, Mathew J., and Michael P. Taylor 2013b. Caudal pneumaticity and pneumatic hiatuses in the sauropod dinosaurs Giraffatitan and Apatosaurus. PLOS ONE 8(10):e78213. 14 pages. doi: 10.1371/journal.pone.0078213

- Witmer, Lawrence M. 1997. The Evolution of the antorbital cavity of archosaurs: a study in soft-tissue reconstruction in the fossil record with an analysis of the function of pneumaticity. Journal of Vertebrate Paleontology 17(S1):1-76. doi:10.1080/02724634.1997.10011027

- Yates, Adam M., Mathew J. Wedel and Matthew F. Bonnan. 2012. The early evolution of postcranial skeletal pneumaticity in sauropodomorph dinosaurs. Acta Palaeontologica Polonica 57(1):85-100. 